\title{
Low collision energy fragmentation in structure-specific glycoproteomics analysis
}

Miloslav Sanda ${ }^{\mathrm{a}, \mathrm{c}}$, Julius Benicky ${ }^{\mathrm{a}, \mathrm{c},}$ Radoslav Goldman ${ }^{\mathrm{a}, \mathrm{b}, \mathrm{c}}$

a Department of Oncology, Lombardi Comprehensive Cancer Center, Georgetown University.

Washington, D.C. 20057, b Department of Biochemistry and Molecular \& Cellular Biology, Georgetown University, Washington, DC, 20057, ${ }^{\mathrm{c}}$ Clinical and Translational Glycoscience Research Center, Georgetown University, Washington, DC, 20057

Supplemental informations 
1. Supplemental Table 1 (Glycoproteins carrying LacdiNAc structure identified in the secretome of HEK293 cells.)

2. Supplemental Figure 1 (Fragmentation spectra of sulfate-containing biantennary LacdiNAc structure recorded under low collision energy (NCE 10) with a specific sulfo-HexNAc oxonium ion $(\mathrm{m} / \mathrm{z} 284.04)$

3. Supplemental Figure 2 (Oxonium ions in the HCD spectra of the Cochlin glycopeptide ENYSSVDANGIQSQMLSQR carrying the biantennary fucosylated LacdiNAc N-glycan. Fragmentation at $3 \operatorname{NCE}(15,20$, and 35) shows the CE-dependent differences in the intensities of ions specific to the structural motifs of fucosylated LacdiNAc (m/z 553.2), LacdiNAc (m/z 407.2), and GalNAc (m/z 186.1).)

4. Supplemental Figure 3 (Oxonium ions in the HCD spectra of Carboxypeptidase E glycopeptide DLQGNPIANATISVEGIDHDVTSAK carrying the monosialylated biantennary LacdiNAc/LacNac N-glycan. Fragmentation at $3 \mathrm{NCE}(15,20$, and 35$)$ shows the CE-dependent differences in the intensities of SialoLacdiNAc (m/z 698.3), Sialo LacNAc (m/z 657.2), LacdiNAc (m/z 407.2), LacNAc (m/z 366.1), and GalNAc (m/z 186.1) structure specific ions.)

5. Supplemental Figure 4 (High collision enery spectra of core-fucosylated biantennary haptoglobin glycopeptide) shows confirmation of core fucosylation by presence of core fucosylated fragments m/z: $1073.07(2+), 1255.13(2+), 1336.16(2+)$ and $1417.69(2+)$.

6. Glycopeptide analysis using Pasef DDA nano LC-MS/MS on the timsTOF Pro 
Supplemental Table 1

Glycoproteins carrying LacdiNAc structure identified in the secretome of HEK293 cells.

\begin{tabular}{|l|l|}
\hline Accession number & Protein name \\
\hline Q03167 & Transforming growth factor beta receptor type 3 \\
\hline P01033 & Metalloproteinase inhibitor 1 \\
\hline O60911 & Cathepsin L2 \\
\hline P35556 & Fibrillin-2 \\
\hline Q02487 & Desmocollin-2 \\
\hline Q8WX77 & Insulin-like growth factor-binding protein-like 1 \\
\hline P50897 & Palmitoyl-protein thioesterase 1 \\
\hline O43405 & Cochlin \\
\hline Q16769 & Glutaminyl-peptide cyclotransferase \\
\hline Q08380 & Galectin-3-binding protein \\
\hline P10909 & Clusterin \\
\hline Q9HAT2 & Sialate O-acetylesterase \\
\hline Q8N2S1 & Latent-transforming growth factor beta-binding protein 4 \\
\hline P41271 & Neuroblastoma suppressor of tumorigenicity 1 \\
\hline O75493 & Carbonic anhydrase-related protein 11 \\
\hline P15151 & Poliovirus receptor \\
\hline P36955 & Pigment epithelium-derived factor \\
\hline P16870 & Carboxypeptidase E \\
\hline
\end{tabular}




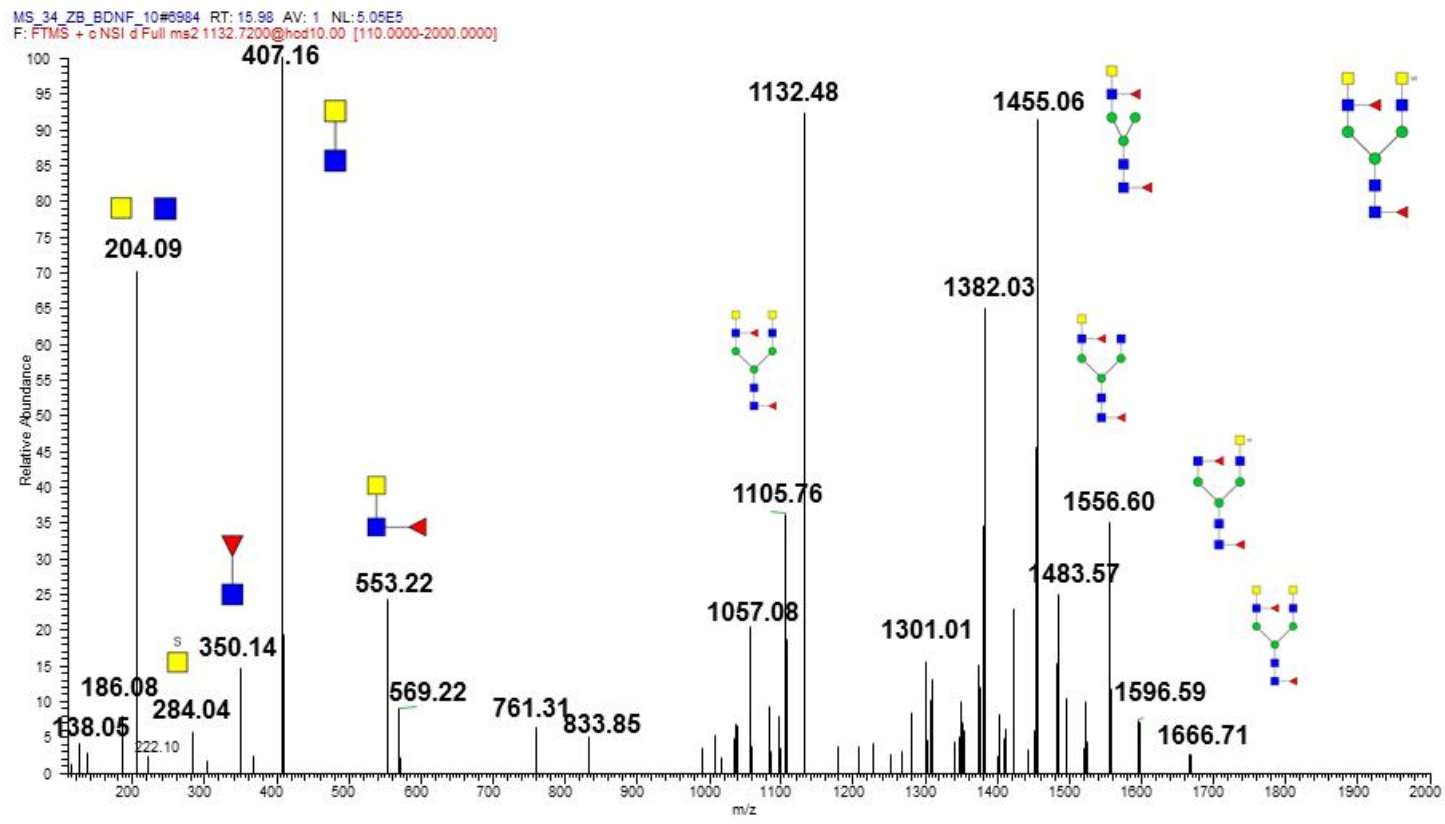

Supplemental Figure 1:

Fragmentation spectra of sulfate-containing biantennary LacdiNAc structure recorded under low collision energy (NCE 10) with a specific sulpho-HexNAc oxonium ion (m/z 284.04) 


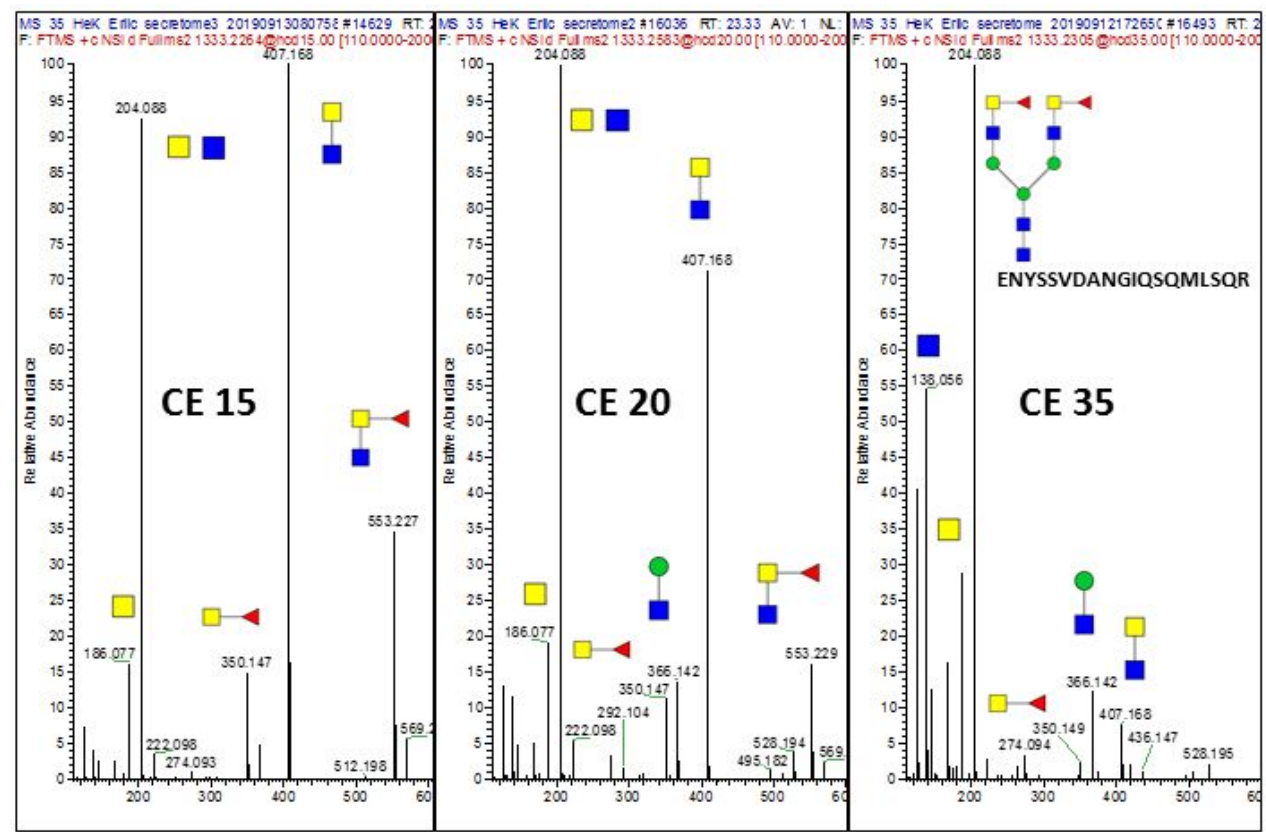

Supplemetal Figure 2:

Oxonium ions in the HCD spectra of the Cochlin glycopeptide ENYSSVDANGIQSQMLSQR carrying the biantennary fucosylated LacdiNAc N-glycan. Fragmentation at 3 NCE $(15,20$, and 35$)$ shows the CE-dependent differences in the intensities of ions specific to the structural motifs of fucosylated LacdiNAc (m/z 553.2), LacdiNAc (m/z 407.2), and GalNAc (m/z 186.1). 


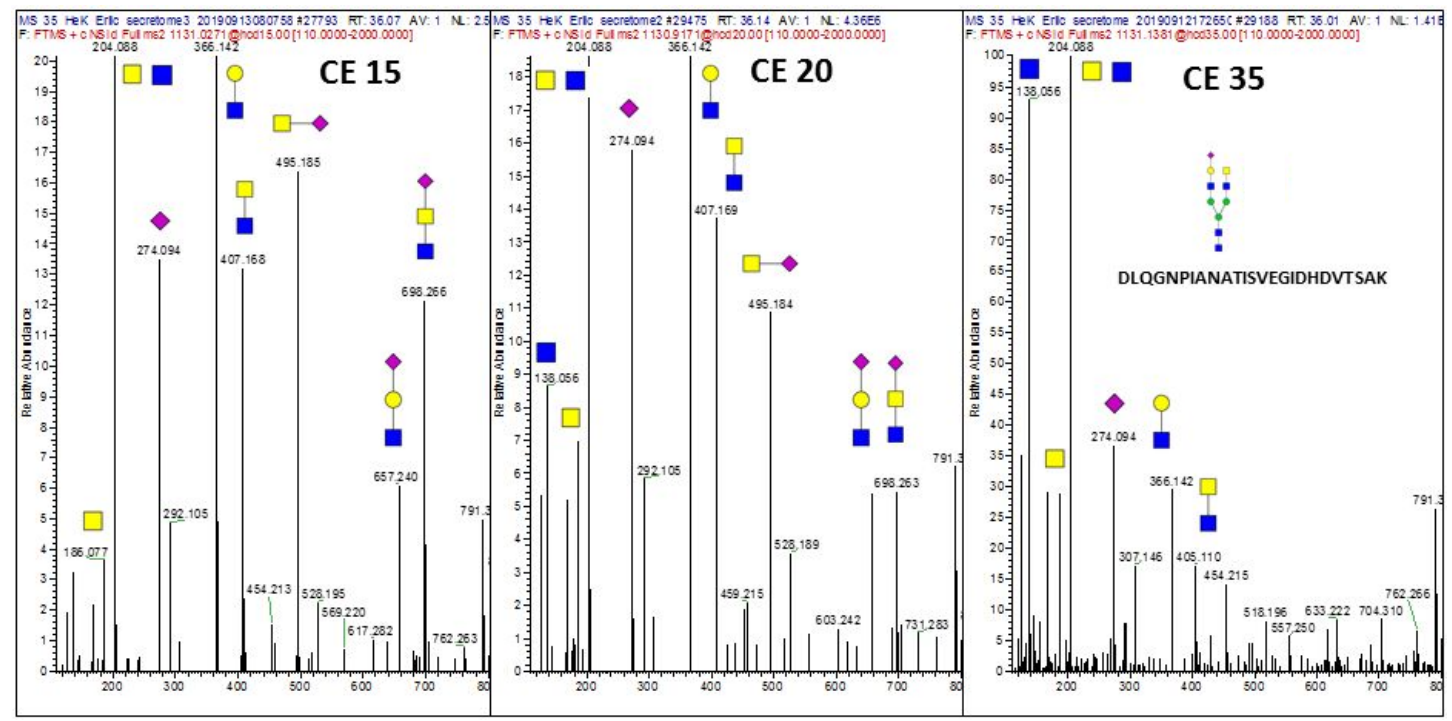

Supplemetal Figure 3:

Oxonium ions in the HCD spectra of Carboxypeptidase E glycopeptide DLQGNPIANATISVEGIDHDVTSAK carrying the monosialylated biantennary LacdiNAc/LacNac Nglycan. Fragmentation at $3 \operatorname{NCE}(15,20$, and 35) shows the CE-dependent differences in the intensities of SialoLacdiNAc (m/z 698.3), Sialo LacNAc (m/z 657.2), LacdiNAc (m/z 407.2), LacNAc (m/z 366.1), and GalNAc (m/z 186.1) structure specific ions. 


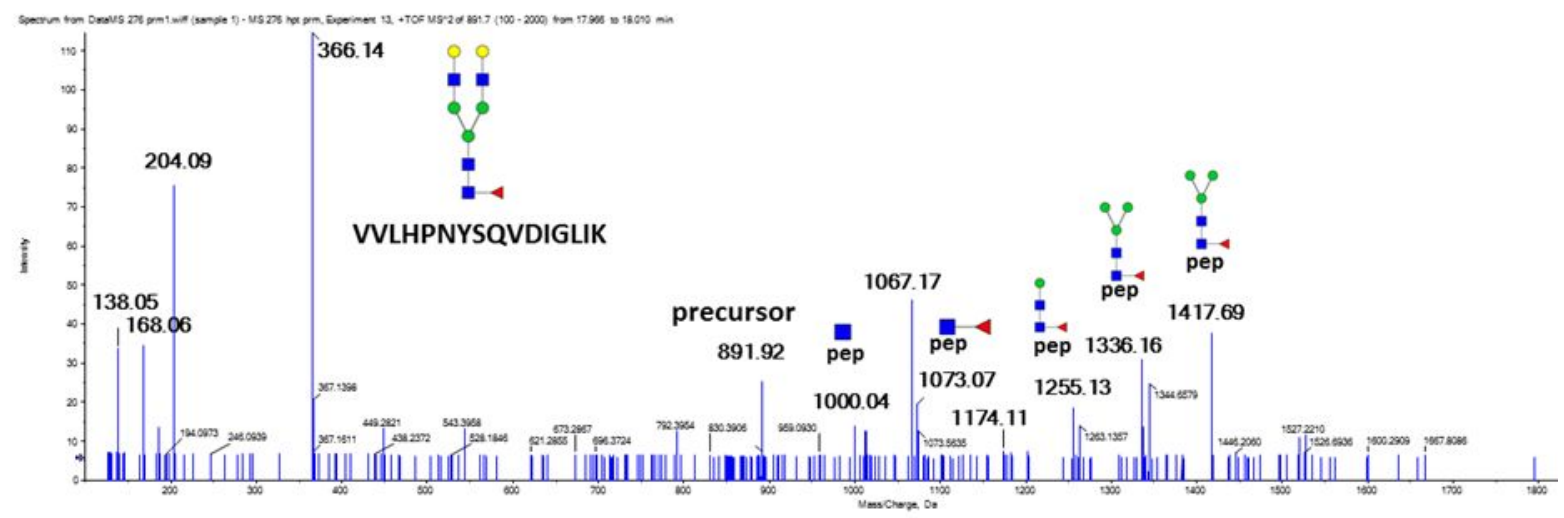

Supplemental Figure 4:

High collision enery spectra of core-fucosylated biantennary haptoglobin glycopeptide) shows confirmation of core fucosylation by presence of core fucosylated fragments m/z: $1073.07(2+)$, $1255.13(2+), 1336.16(2+)$ and $1417.69(2+)$. 
Glycopeptide analysis using Pasef DDA nano LC-MS/MS on the timsTOF Pro

Digested proteins were separated using a 120-minute ACN gradient on a $250 \mathrm{~mm}$ x $75 \mu \mathrm{m}$ C18 Aurura column (lonopticks, Australia) at a flow rate of $0.4 \mu \mathrm{l} / \mathrm{min}$. In brief, peptide and glycopeptide separation was achieved by a $100 \mathrm{~min}$ acetonitrile gradient at a flow rate of 0.4 $\mu \mathrm{l} / \mathrm{min}$ : 0-60 min 0-15\% B (0.1\% formic acid in ACN), 60-90 min 15-23\% B; $90-100$ min $23-35 \%$; 100-110 $\min 35-80 \%$ B; $110-120$ min 80\% B. Glycopeptides were analyzed using timsTOF Pro mass spectrometer with the electrospray ionization voltage at $1600 \mathrm{~V}$ and the capillary temperature at $180^{\circ} \mathrm{C}$. MS1 and MS2 scans were performed over $\mathrm{m} / \mathrm{z} 100-1700$. timsTOF Pro mass spectrometer was operated in PASEF mode over the mobility range $0.75-1.51 / \mathrm{k} 0$, selected precursors for MS2 with charge state 2-7, and dynamic exclusion $0.4 \mathrm{~min}$. MS2 spectra were acquired using stepped collision energy where two MS2 spectra were acquired for each precursor. 